\title{
Predictivity of clinical, laboratory and imaging findings in diagnostic definition of palpable thyroid nodules. A multicenter prospective study
}

\author{
Maria Grazia Chiofalo ${ }^{1}$ - Simona Signoriello ${ }^{2}$ - Franco Fulciniti ${ }^{1,9}$ - Nicola Avenia $^{3}$ - Serenella Ristagno ${ }^{4}$. \\ Celestino Pio Lombardi ${ }^{5}$. Angelo Nicolosi ${ }^{6}$ - Maria Rosa Pelizzo ${ }^{7}$. Giuliano Perigli ${ }^{8}$. Andrea Polistena ${ }^{3}$. \\ Vincenzo Panebianco ${ }^{4} \cdot$ Rocco Bellantone ${ }^{5} \cdot$ Pietro Giorgio Calo $^{6} \cdot$ Isabella Merante Boschin ${ }^{7} \cdot$ Benedetta Badii $^{8}$. \\ Massimo Di Maio ${ }^{1,10} \cdot$ Ciro Gallo $^{2} \cdot$ Francesco Perrone $^{1} \cdot$ Luciano Pezzullo $^{1}{ }^{1}$
}

Received: 18 October 2017 / Accepted: 4 March 2018 / Published online: 22 March 2018

(c) The Author(s) 2018

\begin{abstract}
Purpose To assess the role of clinical, biochemical, and morphological parameters, as added to cytology, for improving presurgical diagnosis of palpable thyroid nodules.

Methods Patients with a palpable thyroid nodule were eligible if surgical intervention was indicated after a positive or suspicious for malignancy FNAC (TIR 4-5 according to the 2007 Italian SIAPEC-IAP classification), or two inconclusive FNAC at a $\geq 3$ months interval, or a negative FNAC associated with one or more risk factor. Reference standard was histological malignancy diagnosis. Likelihood ratios of malignancy, sensitivity, specificity, negative (NPV), and positive predictive value (PPV) were described. Multiple correspondence analysis (MCA) and logistic regression were applied.

Results Cancer was found in 433/902 (48\%) patients. Considering TIR4-5 only as positive cytology, specificity, and PPV were high (94 and 91\%) but sensitivity and NPV were low (61 and 72\%); conversely, including TIR3 among positive, sensitivity and NPV were higher ( 88 and $82 \%$ ) while specificity and PPV decreased (52 and 63\%). Ultrasonographic size $\geq 3 \mathrm{~cm}$ was independently associated with benignity among TIR2 cases (OR of malignancy $0.37,95 \%$ CI $0.18-0.78$ ). In TIR3 cases the hard consistency of small nodules was associated with malignity (OR: $3.51,95 \%$ CI 1.84-6.70, $p<0.001$ ), while size alone, irrespective of consistency, was not diagnostically informative. No other significant association was found in TIR2 and TIR3.

Conclusions The combination of cytology with clinical and ultrasonographic parameters may improve diagnostic definition of palpable thyroid nodules. However, the need for innovative diagnostic tools is still high.
\end{abstract}

These authors contributed equally: Ciro Gallo, Francesco Perrone, Luciano Pezzullo.

Electronic supplementary material The online version of this article (https://doi.org/10.1007/s12020-018-1577-5) contains supplementary material, which is available to authorized users.

Luciano Pezzullo

1.pezzullo@istitutotumori.na.it

1 Istituto Nazionale per lo studio e la Cura dei Tumori, Fondazione G. Pascale, IRCCS, Napoli, Italy

2 Dipartimento di Salute Mentale e Fisica e Medicina Preventiva, Università della Campania Luigi Vanvitelli, Napoli, Italy

3 Endocrinochirurgia, Università di Perugia, Terni, Italy

4 Endocrinochirurgia, Ospedale di Taormina, Taormina, Italy

5 Chirurgia Endocrina, Policlinico Gemelli, Università Cattolica del Sacro Cuore di Roma, Rome, Italy

6 Dipartimento di Scienze Chirurgiche, Università di Cagliari, Cagliari, Italy

7 Patologia Speciale Chirurgica, Azienda Ospedaliera Universitaria di Padova, Padova, Italy

8 Endocrinochirurgia e Chirurgia Miniinvasiva, Azienda Ospedaliera Universitaria Careggi, Firenze, Italy

9 Present address: Istituto Cantonale di Patologia, Locarno, Switzerland

10 Present address: Università di Torino, Turin, Italy 
Keywords Thyroid nodules $\cdot$ Cytology $\cdot$ Prospective observational trial $\cdot$ Multiple correspondence analysis $\cdot$ Diagnostic accuracy

\section{Introduction}

The discovery rate of thyroid nodules has widely risen in last decades, mainly due to the detection of clinically unapparent lesions by means of ultrasonography (US) [1]. This clearly emerged by an old prospective study comparing palpation and US in a population of subjects without known thyroid disease [2]. Authors reported an US prevalence as high as $67 \%$, whereas palpable nodules were detected in only $21 \%$ of the cohort. However, the actual clinical utility of discovering such a quantity of nonpalpable nodules seems to be poor as the risk of malignancy is negligible [3]. Palpable thyroid nodules are reported in approximately $4-7 \%$ of the population $[4,5]$. In this setting, the rate of malignancy is more relevant, being estimated from 8 to $16 \%$ [6, 7]. Therefore, palpable thyroid lesions may be considered as a separate clinical entity requiring a specific diagnostic approach.

To date, several guidelines on the management of thyroid nodules have been published, but a clear distinction between palpable and non palpable lesions has never been performed [6, 8-14]. Furthermore, a number of clinically relevant topics are still debated, and need to be addressed by additional studies. The areas of uncertainty concerns, for instance, the use of ultrasound scan criteria to assess cancer risk, the indication for fine-needle aspiration cytology (FNAC), the new diagnostic and therapeutic techniques, including molecular markers, and the optimal follow-up strategy for patients with benign thyroid nodules.

The evaluation of thyroid nodules includes clinical examination, ultrasound, fine needle aspiration cytology. Currently, FNAC is considered the most accurate test, for evaluation of thyroid nodule due to the high sensitivity $(83 \%)$ and specificity $(92 \%)[15,16]$.

The available literature about the diagnostic accuracy of FNAC, however, is based almost exclusively on retrospective studies performed in cases of positive or indeterminate cytology. Currently, only patients with positive FNAC undergo surgery, thus, for the majority of cases with negative cytology, a histological correlation is not available. The question of false negatives is addressed in several studies [8, 17-23]. A rate of $25 \%$ of false negatives has been reported. Importantly, this percentage is even higher in large palpable nodule $(\geq 4 \mathrm{~cm})[17,24]$. This suggests that FNAC sensitivity may be even more limited in the setting of palpable nodules, where up to a third of cases of malignancy may be missed [22].

Aim of the present study is to assess the role of clinical, biochemical, and morphological parameters as auxiliary tools, added to cytology, for improving pre-surgical diagnosis of palpable thyroid nodules.

\section{Materials and methods}

\section{Study design}

We performed a multicentre prospective observational study to describe the predictive value for diagnosis of malignancy of several clinical, laboratory and radiological parameters, commonly measured in routine clinical practice. The study protocol was approved by the ethical committees at all participating Institutions. Patients were consecutively enrolled at seven participating centers.

\section{Study population}

Patients $\geq 18$ years old were eligible if they had a palpable thyroid nodule, for which surgical intervention was indicated. In details, surgery was indicated when at least one of the following three conditions was present: (1) cytological examination positive or suspicious of malignancy (TIR 4-5 according to the 2007 Italian SIAPEC-IAP classification [25]), (2) two inconclusive cytological examinations with a $\geq 3$ months interval, (3) cytological examination negative for malignant cells (TIR 2-3) associated with at least one major and two minor risk criteria. Major and minor risk criteria were arbitrarily defined by the study protocol. Major risk criteria included clinical (previous neck irradiation), biochemical (calcitonin levels more than twice the upper normal value in absence of renal failure and of pump inhibitors use or positivity at the pentagastrin stimulation test) or ultrasonographic (hypoechogenicity, irregular margins, major diameter $\geq 3 \mathrm{~cm}$, microcalcifications). Minor risk criteria included clinical (hard consistency of the nodule, age $>45$, male gender), biochemical (human thyreoglobulin [HTG] $>2000 \mathrm{ng} / \mathrm{ml}$ ) or ultrasonographic (increase of the major diameter in patients on treatment with thyroxine $\geq 5 \mathrm{~mm}$ if previous diameter was $<1 \mathrm{~cm}$ or $\geq 1 \mathrm{~cm}$ if previous diameter was $\geq 1 \mathrm{~cm}$, intranodular vascularization at Doppler examination).

Patients were excluded in case of concomitant diseases preventing surgery, evidence of locally advanced disease or metastases (lymph nodes or other distant sites). Also patients with non-palpable nodes were excluded. Informed consent was obtained from all individual participants included in the study. 


\section{Study procedures}

Eligible patients underwent FNAC and surgery, independently of the result of cytology. Ultrasonographic evaluation was performed according to participating center practice by experienced radiologists and no independent confirmation was mandated by the protocol.

Cytology interpretation was based on the 2007 Italian SIAPEC-IAP 5-category scale: TIR1: non-diagnostic, inadequate (smears should contain 6 or more groups of at least 10 thyroid follicular cells to be considered adequate); TIR2: benign (consistent with nodular goiter or thyroiditis); TIR3: indeterminate; TIR4: suspicious of malignancy; TIR5: malignant [25]. Palpable nodules $>2 \mathrm{~cm}$ or containing microcalcifications had to be sampled with at least two needle passes. Samples with macroscopic blood contamination had to be repeated immediately, when feasible $[26,27]$. There was no independent revision of cytological and histological diagnosis, according to a pragmatic approach, considering that all participating centers are considered as referral Institutions for thyroid cancer.

\section{Statistical analysis}

Diagnostic performance of FNAC was assessed using likelihood ratio (LR), sensitivity, specificity, negative predictive value (NPV), and positive predictive value (PPV); for all the estimated $95 \%$ confidence intervals were calculated. Reference standard was histological diagnosis (malignant/benign). Three categories of FNAC were used (TIR2, TIR3, and TIR4-5). LR was estimated for each FNAC category. Since the other measures of accuracy (sensitivity, specificity, PPV and NPV) can only be calculated with dichotomous tests, we estimated them twice, considering as positive in turn either TIR4 and TIR5 categories (TIR4-5) or the previous ones combined with the TIR3 category (TIR3-4-5).

Associations of clinical, biochemical and ultrasonographic variables with malignancy were separately evaluated in patients with TIR2 and TIR3 categories. Surgery indeed was mandatory in patients with TIR4 or TIR5 cytological diagnosis and any further information would be irrelevant for clinical decision.

Univariate associations were tested using Pearson's chisquare test and Fisher's exact test, as appropriate. Multivariable analyses were performed by means of multiple logistic regression models.

Since the six ultrasonographic variables were expected to be highly correlated, relationships between them were preliminary investigated with multiple correspondence analysis (MCA) [28]. MCA is a graphical technique that allows to display categories of variables as points in a geometric map of relationship. The plot axes indicate latent variables that are derived from the contribution of the single variables. Proximities of categories in the two-dimensional plot suggest possible associations between them. As a general rule the distance between two points may be roughly interpreted as the correlation among them although findings must also be read in the light of the quantitative contributions of the categories to the axes $\left(\eta^{2}\right)$. The percentage of information explained (i.e., the percentage of total variability explained by the two axes of the plot) was measured following Benzécri: higher the percentage better the two-dimensional representation of data [29]. To improve the interpretation of results, the two histology categories (malignant/benign) were depicted on the MCA plot as supplementary variables (i.e., they did not contribute to the axes). As a result of the exploratory MCA the six ultrasonographic variables were combined in two new variables that were entered as covariates in the logistic models.

For all analyses, results were considered statistically significant if the two-tailed $p$-value was 0.05 or less. All analyses were performed with $\mathrm{R}$ software, version 3.0.0 [The R Foundation for Statistical Computing 2013].

\section{Results}

Overall 914 eligible patients were enrolled at seven centers between March 2010 and April 2012. Twelve patients underwent surgery because of two consecutive inconclusive cytological examinations; these patients were excluded from subsequent analysis. Baseline characteristics of the remaining 902 patients are reported in Table 1. Patients were prevalently females (76\%) with a median age of 49 years. Biochemical variables were found positive in a very small number of patients (23 cases overall). FNAC categories were evenly distributed in the study patients $(33 \%$ TIR2, 35\% TIR3 and 32\% TIR4-5). A malignant tumor was present in $48 \%$ of patient, mostly as papillary carcinoma (86\%).

Estimated LRs for each TIR category are reported in Table 2. As expected the two extreme categories (TIR4-5 and TIR2) provided stronger information for or against malignancy, respectively, while the intermediate category (TIR3) was less informative. To estimate the other measures of accuracy (Table 3 ) two $2 \times 2$ contingency tables were derived where the intermediate category TIR3 was alternatively considered negative (and pooled with TIR2) or positive (and pooled with TIR4-5). When a "positive" result was limited to TIR4-5 category, sensitivity was equal to $61 \%$ (i.e., there was a false negative rate of $39 \%$ ) and specificity was equal to $94 \%$, meaning that only $6 \%$ of false positive patients were found. Accordingly PPV was high. Conversely, when the indeterminate TIR3 category was combined with TIR4-5 in the definition of 'positive' result, 
Table 1 Baseline characteristics of the study patients

\begin{tabular}{|c|c|}
\hline Variable & $N=902$ \\
\hline Age at diagnosis years, median (range) & $49(18-87)$ \\
\hline \multicolumn{2}{|l|}{ Clinical variables } \\
\hline Previous neck irradiation & $5(1 \%)$ \\
\hline Hard consistency at palpation & $428(47 \%)$ \\
\hline Age $>45$ years & $553(61 \%)$ \\
\hline Male gender & $213(24 \%)$ \\
\hline \multicolumn{2}{|l|}{ Biochemical variables } \\
\hline Serum calcitonin level $>2 \mathrm{UNL}$ & $12(1 \%)$ \\
\hline Positive pentagastrin stimulation test & $3(0 \%)$ \\
\hline Serum thyreoglobulin $>2000 \mathrm{ng} / \mathrm{ml}$ & $8(1 \%)$ \\
\hline \multicolumn{2}{|l|}{ Ultrasonography variables } \\
\hline Hypoechogenicity & $719(80 \%)$ \\
\hline Irregular margins & $348(39 \%)$ \\
\hline Size $\geq 3 \mathrm{~cm}$ & $428(47 \%)$ \\
\hline Microcalcifications & $379(42 \%)$ \\
\hline Increase in nodule volume & $216(24 \%)$ \\
\hline Intranodular vascularization & $624(69 \%)$ \\
\hline \multicolumn{2}{|l|}{ Fine-needle aspiration category } \\
\hline TIR2 & $295(33 \%)$ \\
\hline TIR3 & $315(35 \%)$ \\
\hline TIR4-5 & $292(32 \%)$ \\
\hline Malignant histology & $435(48 \%)$ \\
\hline Papillary carcinoma & $374(41 \%)$ \\
\hline Follicular carcinoma & $46(5 \%)$ \\
\hline Anaplastic carcinoma & $5(1 \%)$ \\
\hline Medullary carcinoma & $8(1 \%)$ \\
\hline Missing & $2(0 \%)$ \\
\hline \multicolumn{2}{|l|}{ City of the participating center } \\
\hline Terni & $210(23 \%)$ \\
\hline Taormina & $196(22 \%)$ \\
\hline Roma & $124(14 \%)$ \\
\hline Napoli & $114(13 \%)$ \\
\hline Cagliari & $108(12 \%)$ \\
\hline Padova & $81(9 \%)$ \\
\hline Firenze & $69(8 \%)$ \\
\hline
\end{tabular}

sensitivity increased to $88 \%$ (i.e., $12 \%$ of patients with malignancy were found negative), but specificity decreased to an unacceptably value of $52 \%$, meaning that $48 \%$ patients without malignancy were found positive. Accordingly PPV value was only $63 \%$.

Results of univariate analyses are summarized in the supplementary Table online separately for FNAC negative (TIR2) and indeterminate (TIR3) subjects. In the TIR2 subjects, size $\geq 3 \mathrm{~cm}$ was significantly associated with the absence of malignancy while no statistically significant association was found for any other variable. Within the TIR3 category, three variables were found to be associated
Table 2 Diagnostic accuracy of fine-needle aspiration (FNA) category

\begin{tabular}{llllll}
\hline & \multicolumn{2}{l}{ Histology } & & & \\
\cline { 2 - 4 } FNA category & Malignant & Benign & Total & LR & (CI 95\%) \\
\hline TIR2 & 53 & 242 & 295 & 0.23 & $(0.18-0.31)$ \\
TIR3 & 116 & 199 & 315 & 0.63 & $(0.52-0.76)$ \\
TIR4-5 & 266 & 26 & 292 & 10.98 & $(7.5-16.08)$ \\
Total & 435 & 467 & 902 & & \\
\hline
\end{tabular}

Likelihood ratios for malignancy

Table 3 Sensitivity, specificity and predictive values for malignancy ( $95 \%$ confidence intervals)

\begin{tabular}{lllll}
\hline $\begin{array}{l}\text { Definition of test } \\
\text { outcome }\end{array}$ & Sensitivity & Specificity & PPV & NPV \\
\hline Positive: TIR4-5 & $61 \%$ & $94 \%$ & $91 \%$ & $72 \%$ \\
Negative: TIR2-3 & $(56-66 \%)$ & $(92-96 \%)$ & $(87-94 \%)$ & $(69-76 \%)$ \\
Positive: TIR3-4-5 & $88 \%$ & $52 \%$ & $63 \%$ & $82 \%$ \\
Negative: TIR2 & $(84-91 \%)$ & $(47-56 \%)$ & $(59-67 \%)$ & $(77-86 \%)$ \\
\hline
\end{tabular}

$P P V$ positive predictive value, $N P V$ negative predictive value

with histology: hard consistency at palpation and irregular margins were more frequent in presence of malignancy while again size $\geq 3 \mathrm{~cm}$ was more frequently observed when malignancy was absent. Because of the very small prevalence, biochemical variables and previous neck irradiation were no further evaluated in multivariate analyses.

Exploratory MCA was performed separately in FNACTIR2 and FNAC-TIR3 patients. MCA two-dimensional plot in TIR2 patients is depicted in Fig. 1. The first two axes accounted for $83 \%$ of the total variability. The first axis could be mainly interpreted as expression of morphological modifications since it was associated with irregular margins, hypoechogenicity, intranodular vascularization and microcalcifications $\left(\eta^{2}\right.$ equal to $0.67,0.52,0.53,0.29$, respectively). The second axis was strongly associated only with size $\left(\eta^{2}=0.83\right)$. The histology categories, plotted as supplementary variables, were only slightly correlated with the second axis. Following this exploratory analysis, tumor size remained as single variable and a new ultrasonographic variable was derived, morphological modifications, summing up the categories on the same side along the first axis (presence of irregular margins, hypoechogenicity, intranodular vascularization, and microcalcifications). These two ultrasonographic variables were entered into the logistic model as covariates.

MCA two-dimensional plot in TIR3 patients is depicted in Fig. 2. The first two axes accounted for $68 \%$ of the total variability. The two axes may be read as above, and the first axis was associated with irregular margins, hypoechogenicity, intranodular vascularization and microcalcifications $\left(\eta^{2}\right.$ equal to $0.55,0.38,0.48$, and 0.38 , respectively). As above, 


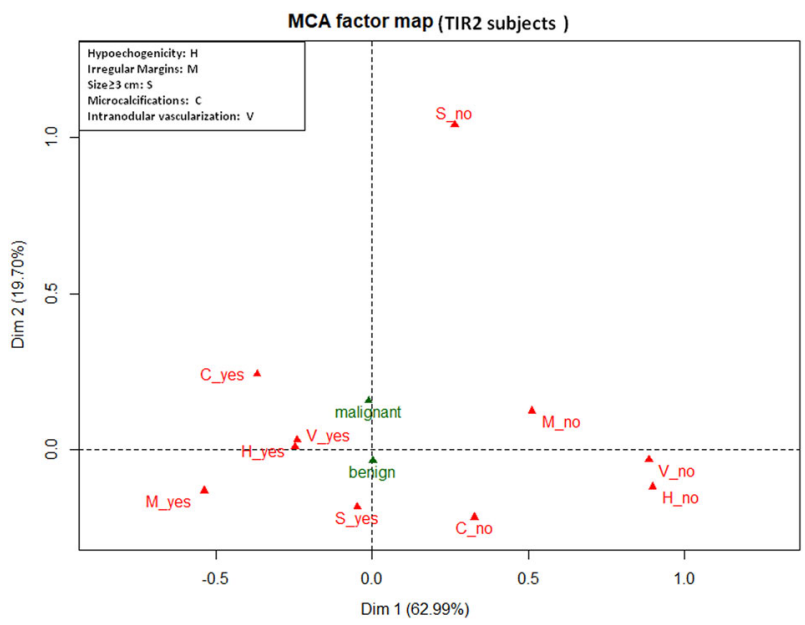

Fig. 1 Multiple correspondence (MCA) two-dimensional plot in TIR2 patients

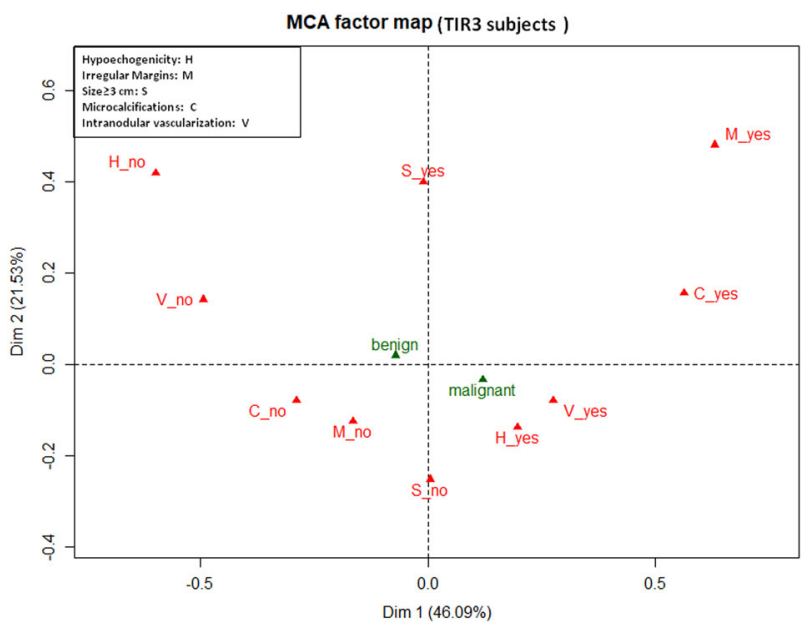

Fig. 2 Multiple correspondence (MCA) two-dimensional plot in TIR3 patients

size was associated with the second axis $\left(\eta^{2}=0.78\right)$. Following this exploratory analysis, the same two ultrasonographic variables were entered into the logistic model as covariates.

Results of the logistic regression analyses are reported in Table 4. The new variable "morphological modifications", ranging from 0 to 4 , was entered in the models as a continuous covariate. As for TIR 2 cases, only size $\geq 3 \mathrm{~cm}$ was statistically associated with benign tumor (OR $0.37,95 \%$ CI $0.18-0.78, p=0.008)$. As for TIR3 cases, interaction between consistency and size was statistically significant ( $p$ $=0.028$ ), therefore we entered into the model three dummy variables of combinations of consistency and size; soft consistency and size $<3 \mathrm{~cm}$ was the reference category. It appeared that hard consistency combined with small size $(<3 \mathrm{~cm})$ was a strong predictor of malignancy (OR 3.51, 95\% CI 1.84-6.70, $p<0.001)$, while the other two
Table 4 Logistic regression analysis

OR $\quad 95 \%$ CI $\quad p$-value

TIR2

Hard consistency at palpation

$\begin{array}{lll}1.16 & 0.58-2.29 & 0.678\end{array}$

Age $>45$ years

$0.66 \quad 0.34-1.28 \quad 0.216$

Male gender

$\begin{array}{lll}1.55 & 0.76-3.16 & 0.224\end{array}$

Morphological modifications ${ }^{\mathrm{a}}$

$1.07 \quad 0.81-1.41 \quad 0.630$

Size $\geq 3$ vs. $<3$

$\begin{array}{lll}0.37 & 0.18-0.78 & \mathbf{0 . 0 0 8}\end{array}$

TIR3

Hard consistency and size $<3 \mathrm{~cm}$ vs. soft $3.51 \quad 1.84-6.70<\mathbf{0 . 0 0 1}$ consistency and size $<3 \mathrm{~cm}$

Soft consistency and size $\geq 3 \mathrm{~cm}$ vs. soft $\quad 0.74 \quad 0.39-1.40 \quad 0.357$ consistency and size $<3 \mathrm{~cm}$

Hard consistency and size $\geq 3 \mathrm{~cm}$ vs. soft $\quad 0.79 \quad 0.35-1.76 \quad 0.563$ consistency and size $<3 \mathrm{~cm}$

Age $>45$ years

$0.91 \quad 0.55-1.51 \quad 0.719$

Male gender

$\begin{array}{lll}1.22 & 0.71-2.10 & 0.470\end{array}$

Morphological modifications ${ }^{\mathrm{a}}$

$1.26 \quad 0.99-1.59 \quad 0.056$

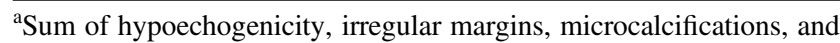
intranodular vascularization (range $0-4$ )

bold significants to $p$-values

Table 5 Distribution of definitive histology by combination of consistency and size of the nodule in TIR3 cases

\begin{tabular}{lll}
\hline & Histology & \\
\cline { 2 - 3 } & Malignant & Benign \\
\hline Hard consistency and size $<3 \mathrm{~cm}$ & $40(68 \%)$ & $19(32 \%)$ \\
Hard consistency and size $\geq 3 \mathrm{~cm}$ & $16(33 \%)$ & $29(67 \%)$ \\
Soft consistency and size $<3 \mathrm{~cm}$ & $34(31 \%)$ & $77(69 \%)$ \\
Soft consistency and size $\geq 3 \mathrm{~cm}$ & $20(27 \%)$ & $55(73 \%)$ \\
Total & $116(37 \%)$ & $199(63 \%)$ \\
\hline
\end{tabular}

categories were not statistically significant. For a better understanding the distribution of malignant histology according to combination of size and consistency at palpation is reported in Table 5. The subgroup with the higher percentage of malignancy is the one with hard consistency at palpation and low nodule size.

\section{Discussion}

To the best of our knowledge, this is the first study specifically focusing on palpable thyroid nodules, considered as a separate clinical entity, and trying to provide specific indications about the diagnostic work-up of this clinical setting.

A crucial feature of our paper is that the study population included patients with palpable thyroid nodules who meet surgical criteria, including both cytology results and clinical parameters considered as risk factors of malignancy. This 
explains why nearly a half of the study subjects, therefore much more than expected from a general cohort of patients with palpable nodules, were diagnosed with malignant disease at histology. By contrast, this also means that more than a half of patients advised to surgery basing on a presurgical cancer risk assessment actually harbor benign disease, thus indicating that current management of palpable thyroid nodules needs to be improved. Another relevant consequence of the composition of the cohort is that the study cannot provide information about FNAC accuracy, as taken alone. Indeed, only those subjects with benign cytology carrying clinical risk factors of malignancy have been included, and this represents a bias.

Therefore, the main aim of the study was to verify the diagnostic accuracy, as defined by the capability of identifying patients with cancer, of the combination between cytology and clinical parameters. Methodologically, a crucial point was the definition of 'positive' cytological result. Indeed, the TIR3 category is by definition a gray zone. Furthermore, the recently introduced TIR3 subcategories are not available as still not used in clinical practice at the time of the study, and this represents a limit of our analysis [30-32]. We therefore performed 2 separate analyses considering the TIR3 cytology as a negative (together with TIR2) and positive (together with TIR 4-5) result, respectively (Table 3.) When considering TIR 2-3 as the negative cytological result, the sensitivity was only $61 \%$, which means that nearly $40 \%$ of malignant tumors were missed. By contrast, when TIR2 alone was considered as the only negative cytological category, the sensitivity rose to $88 \%$, determining a negative predictive value of $82 \%$. This means that the combination between TIR2 cytology and presurgical clinical assessment, as performed in this protocol, was related to a false negatives rate of only $12 \%$, which was far more less than expected basing on literature data (as previously indicated).

In order to characterize specific diagnostic impact of the assessed clinical variables, we analyzed the relationship of each of them with final histology into the categories TIR2 and TIR3, as for TIR4-5 ones surgery is mandatory and any further information would be irrelevant for clinical decisions. Around $38 \%$ of cases categorized as TIR 2 or TIR3 actually had cancer, consistent with some literature claims [22].

In both TIR 2 and three categories, an independent relationship was found between large nodule size and benign histology. This implies that the likelihood of malignancy decreased in those palpable nodules with TIR2-3 cytology when the diameter is $\geq 3 \mathrm{~cm}$. Therefore, basing on previous analysis, a TIR 2 cytology from a large palpable nodule is related to low risk of false negativity. However, our finding that large tumors are associated with a lower risk of malignancy is not consistent with some reports that might be influenced by the fact only a subset of patients underwent preoperative FNAC [33, 34].

In the TIR3 category, hard consistency at palpation in small nodules was associated with malignancy and this may represent a useful indication for the management of palpable nodules with indeterminate cytology letting clinicians to choose thyroidectomy instead of follow-up.

The major strength of the present study is that the sample size is quite large, as derived from institutions with a high volume and high experience in the management of thyroid cancer, collected prospectively, and with definitive histological examination as gold standard for definition of performance of FNAC.

This study has, of course, also several limitations. As aforementioned, our population is not representative of the whole population of patients with palpable thyroid nodules, because only patients for whom surgery was indicated were included. As aforementioned, the recently introduced TIR3 subcategories were not used. Another issue is represented by the concordance between different pathologists [35]. Finally, inter-observer variability may also potentially affect ultrasonography [36].

In conclusion, the combination between cytology and assessment of clinical parameters (including clinics and US findings) represents a feasible diagnostic approach for palpable thyroid nodules. However, the need for innovative diagnostic tools for malignancy, driving decision making in clinical practice, is still high.

Acknowledgements We thank the following peole working at Istituto Nazionale per lo studio e la Cura dei Tumori, Fondazione Pascale, IRCCS, Napoli: Franca La Vecchia, Mariella Staiano, Maria Pia Curcio, Anna Cipolletta Campanile and Gabriella Malzone for collaboration to cytology diagnosis; Federica Hauber and Michele Gallo for collaboration with Thyroid Surgery Unit; Manuela Florio, Giuliana Canzanella, Federika Crudele, Cristiana De Luca, Giovanni de Matteis, Fiorella Romano, Alfonso Savio.

Funding This study had no funding and was performed with institutional support of the coordinating institution (Istituto Nazionale per lo studio e la Cura dei Tumori, Fondazione G.Pascale, IRCCS, Napoli; Italy).

\section{Compliance with ethical standards}

Conflict of interest The authors declare that they have no conflict of interest.

Ethical approval This study has been approved by ethics committee at all participating centers and all procedures were in accordance with the ethical standards of the ethical committees and of the 1964 Declaration of Helsinki and its later amendments.

Informed consent Informed consent was obtained from all individual patients included in the study. 
Open Access This article is distributed under the terms of the Creative Commons Attribution 4.0 International License (http://crea tivecommons.org/licenses/by/4.0/), which permits unrestricted use, distribution, and reproduction in any medium, provided you give appropriate credit to the original author(s) and the source, provide a link to the Creative Commons license, and indicate if changes were made.

\section{References}

1. D.S. Dean, H. Gharib, Epidemiology of thyroid nodules. Best Pract. Res. Clin. Endocrinol. Metab. 22(6), 901-911 (2008). https://doi.org/10.1016/j.beem.2008.09.019

2. S. Ezzat, D.A. Sarti, D.R. Cain, G.D. Braunstein, Thyroid incidentalomas. Prevalence by palpation and ultrasonography. Arch. Intern. Med. 154(16), 1838-1840 (1994)

3. E. Papini, R. Guglielmi, A. Bianchini, A. Crescenzi, S. Taccogna, F. Nardi, C. Panunzi, R. Rinaldi, V. Toscano, C.M. Pacella, Risk of malignancy in nonpalpable thyroid nodules: predictive value of ultrasound and color-Doppler features. J. Clin. Endocrinol. Metab. 87(5), 1941-1946 (2002). https://doi.org/10.1210/jcem.87.5.8504

4. W.M. Tunbridge, D.C. Evered, R. Hall, D. Appleton, M. Brewis, F. Clark, J.G. Evans, E. Young, T. Bird, P.A. Smith, The spectrum of thyroid disease in a community: the Whickham survey. Clin. Endocrinol. 7(6), 481-493 (1977)

5. J.B. Vander, E.A. Gaston, T.R. Dawber, The significance of nontoxic thyroid nodules. Final report of a 15-year study of the incidence of thyroid malignancy. Ann. Intern. Med. 69(3), 537-540 (1968)

6. D.S. Cooper, G.M. Doherty, B.R. Haugen, R.T. Kloos, S.L. Lee, S.J. Mandel, E.L. Mazzaferri, B. McIver, F. Pacini, M. Schlumberger, S.I. Sherman, D.L. Steward, R.M. Tuttle, Revised American Thyroid Association management guidelines for patients with thyroid nodules and differentiated thyroid cancer. Thyroid 19(11), 1167-1214 (2009). https://doi.org/10.1089/thy.2009.0110

7. L. Yassa, E.S. Cibas, C.B. Benson, M.C. Frates, P.M. Doubilet, A. A. Gawande, F.D. Moore Jr., B.W. Kim, V. Nose, E. Marqusee, P. R. Larsen, E.K. Alexander, Long-term assessment of a multidisciplinary approach to thyroid nodule diagnostic evaluation. Cancer 111(6), 508-516 (2007). https://doi.org/10.1002/cncr.23116

8. H. Gharib, E. Papini, R. Paschke, Thyroid nodules: a review of current guidelines, practices, and prospects. Eur. J. Endocrinol. 159(5), 493-505 (2008). https://doi.org/10.1530/EJE-08-0135

9. H. Gharib, E. Papini, R. Paschke, D.S. Duick, R. Valcavi, L. Hegedus, P. Vitti, American Association of Clinical Endocrinologists, Associazione Medici Endocrinologi, and European Thyroid Association medical guidelines for clinical practice for the diagnosis and management of thyroid nodules. J. Endocrinol. Invest 33(5 Suppl), 1-50 (2010)

10. H. Gharib, E. Papini, R. Valcavi, H.J. Baskin, A. Crescenzi, M.E. Dottorini, D.S. Duick, R. Guglielmi, C.R. Hamilton Jr., M.A. Zeiger, M. Zini, American Association of Clinical Endocrinologists and Associazione Medici Endocrinologi medical guidelines for clinical practice for the diagnosis and management of thyroid nodules. Endocr. Pract. 12(1), 63-102 (2006). https://doi.org/10. 4158/EP.12.1.63

11. B.R. Haugen, E.K. Alexander, K.C. Bible, G.M. Doherty, S.J. Mandel, Y.E. Nikiforov, F. Pacini, G.W. Randolph, A.M. Sawka, M. Schlumberger, K.G. Schuff, S.I. Sherman, J.A. Sosa, D.L. Steward, R.M. Tuttle, L. Wartofsky, 2015 American thyroid association management guidelines for adult patients with thyroid nodules and differentiated thyroid cancer: the American thyroid association guidelines task force on thyroid nodules and differentiated thyroid cancer. Thyroid 26(1), 1-133 (2016). https://doi. org/10.1089/thy. 2015.0020
12. F. Pacini, M. Schlumberger, H. Dralle, R. Elisei, J.W. Smit, W. Wiersinga, European consensus for the management of patients with differentiated thyroid carcinoma of the follicular epithelium. Eur. J. Endocrinol. 154(6), 787-803 (2006). https://doi.org/10. 1530/eje. 1.02158

13. P. Perros, K. Boelaert, S. Colley, C. Evans, R.M. Evans, G. Gerrard Ba, J. Gilbert, B. Harrison, S.J. Johnson, T.E. Giles, L. Moss, V. Lewington, K. Newbold, J. Taylor, R.V. Thakker, J. Watkinson, G.R. Williams, Guidelines for the management of thyroid cancer. Clin. Endocrinol. 81(Suppl 1), 1-122 (2014). https://doi.org/10.1111/cen.12515

14. M.B. Pitman, J. Abele, S.Z. Ali, D. Duick, T.M. Elsheikh, R.B. Jeffrey, C.N. Powers, G. Randolph, A. Renshaw, L. Scoutt, Techniques for thyroid FNA: a synopsis of the National Cancer Institute Thyroid Fine-Needle Aspiration State of the Science Conference. Diagn. Cytopathol. 36(6), 407-424 (2008). https:// doi.org/10.1002/dc.20829

15. A. Kessler, H. Gavriel, S. Zahav, M. Vaiman, N. Shlamkovitch, S. Segal, E. Eviatar, Accuracy and consistency of fine-needle aspiration biopsy in the diagnosis and management of solitary thyroid nodules. Isr. Med. Assoc. J. 7(6), 371-373 (2005)

16. J.L. Morgan, J.W. Serpell, M.S. Cheng, Fine-needle aspiration cytology of thyroid nodules: how useful is it? ANZ J. Surg. 73(7), 480-483 (2003)

17. J. Aguilar, J.M. Rodriguez, B. Flores, J. Sola, A. Bas, T. Soria, P. Ramirez, P. Parrilla, Value of repeated fine-needle aspiration cytology and cytologic experience on the management of thyroid nodules. Otolaryngol. Head Neck Surg. 119(1), 121-124 (1998)

18. K.A. Al-Hureibi, A.A. Al-Hureibi, Y.A. Abdulmughni, S.M. Aulaqi, M.S. Salman, E.M. Al-Zooba, The diagnostic value of fine needle aspiration cytology in thyroid swellings in a university hospital, Yemen. Saudi Med J. 24(5), 499-503 (2003)

19. N. Leonard, D.H. Melcher, To operate or not to operate? The value of fine needle aspiration cytology in the assessment of thyroid swellings. J. Clin. Pathol. 50(11), 941-943 (1997)

20. A. Lucas, M. Llatjos, I. Salinas, J. Reverter, E. Pizarro, A. Sanmarti, Fine-needle aspiration cytology of benign nodular thyroid disease. Value re-aspiration. Eur. J. Endocrinol. 132(6), 677-680 (1995)

21. S.H. Merchant, R. Izquierdo, K.K. Khurana, Is repeated fineneedle aspiration cytology useful in the management of patients with benign nodular thyroid disease? Thyroid 10(6), 489-492 (2000)

22. Y.Y. Tee, A.J. Lowe, C.A. Brand, R.T. Judson, Fine-needle aspiration may miss a third of all malignancy in palpable thyroid nodules: a comprehensive literature review. Ann. Surg. 246(5), 714-720 (2007). https://doi.org/10.1097/SLA.0b013e3180f61adc

23. G.P. Yeoh, K.W. Chan, The diagnostic value of fine-needle aspiration cytology in the assessment of thyroid nodules: a retrospective 5-year analysis. Hong Kong Med J. 5(2), 140-144 (1999)

24. A. Rios, J.M. Rodriguez, P.J. Galindo, M. Montoya, F.J. Tebar, J. Sola, M. Canteras, P. Parrilla, Utility of fine-needle aspiration for diagnosis of carcinoma associated with multinodular goitre. Clin. Endocrinol. 61(6), 732-737 (2004). https://doi.org/10.1111/j. 1365-2265.2004.02157.x

25. G. Fadda, F. Basolo, A. Bondi, G. Bussolati, A. Crescenzi, O. Nappi, F. Nardi, M. Papotti, G. Taddei, L. Palombini, S.-I.I.C.W. Group, Cytological classification of thyroid nodules. Proposal of the SIAPEC-IAP Italian Consensus Working Group. Pathologica 102(5), 405-408 (2010)

26. A. Bartolazzi, F. Orlandi, E. Saggiorato, M. Volante, F. Arecco, R. Rossetto, N. Palestini, E. Ghigo, M. Papotti, G. Bussolati, M.P. Martegani, F. Pantellini, A. Carpi, M.R. Giovagnoli, S. Monti, V. Toscano, S. Sciacchitano, G.M. Pennelli, C. Mian, M.R. Pelizzo, M. Rugge, G. Troncone, L. Palombini, G. Chiappetta, G. Botti, A. 
Vecchione, R. Bellocco, Galectin-3-expression analysis in the surgical selection of follicular thyroid nodules with indeterminate fine-needle aspiration cytology: a prospective multicentre study. Lancet Oncol. 9(6), 543-549 (2008). https://doi.org/10.1016/ S1470-2045(08)70132-3

27. F. Fulciniti, G. Benincasa, A. Vetrani, L. Palombini, Follicular variant of papillary carcinoma: cytologic findings on FNAB samples-experience with 16 cases. Diagn. Cytopathol. 25(2), 86-93 (2001)

28. M. Greenacre, J. Blasius. Multiple Correspondence Analysis and Related Methods. Statistics in the Social and Behavioral Sciences Series. (Chapman \& Hall/CRC, Boca Raton, FL), 2006)

29. L. Lebart, A. Morineau, M. Piron. Statistique exploratoire multidimensionnelle. (Dunod, Paris), 1995)

30. Z.W. Baloch, V.A. LiVolsi, S.L. Asa, J. Rosai, M.J. Merino, G. Randolph, P. Vielh, R.M. DeMay, M.K. Sidawy, W.J. Frable, Diagnostic terminology and morphologic criteria for cytologic diagnosis of thyroid lesions: a synopsis of the National Cancer Institute Thyroid Fine-Needle Aspiration State of the Science Conference. Diagn. Cytopathol. 36(6), 425-437 (2008). https:// doi.org/10.1002/dc. 20830

31. P.C.A. Cross, T. Giles, S. Johnson, G. Kocjan, D. Poller, T. Stephenson, Guidance on the Reporting of Thyroid Cytology Specimens (The Royal College of Pathologists, London 2016)

32. F. Nardi, F. Basolo, A. Crescenzi, G. Fadda, A. Frasoldati, F. Orlandi, L. Palombini, E. Papini, M. Zini, A. Pontecorvi, P. Vitti,
Italian consensus for the classification and reporting of thyroid cytology. J. Endocrinol. Invest 37(6), 593-599 (2014). https://doi. org/10.1007/s40618-014-0062-0

33. B. Kuru, N.E. Gulcelik, M.A. Gulcelik, H. Dincer, The falsenegative rate of fine-needle aspiration cytology for diagnosing thyroid carcinoma in thyroid nodules. Langenbeck's Arch. Surg. 395(2), 127-132 (2010). https://doi.org/10.1007/s00423-0090470-3

34. S.N. Pinchot, H. Al-Wagih, S. Schaefer, R. Sippel, H. Chen, Accuracy of fine-needle aspiration biopsy for predicting neoplasm or carcinoma in thyroid nodules $4 \mathrm{~cm}$ or larger. Arch. Surg. 144 (7), 649-655 (2009). https://doi.org/10.1001/archsurg.2009.116

35. C. Bellevicine, I. Migliatico, E. Vigliar, N. Serra, G. Troncone, Intra-institutional second opinion diagnosis can reduce unnecessary surgery for indeterminate thyroid FNA: a preliminary report on 34 cases. Cytopathology 28(4), 254-258 (2017). https://doi. org/10.1111/cyt.12431

36. G. Grani, L. Lamartina, V. Ascoli, D. Bosco, F. Nardi, F. D’Ambrosio, A. Rubini, L. Giacomelli, M. Biffoni, S. Filetti, C. Durante, V. Cantisani, Ultrasonography scoring systems can rule out malignancy in cytologically indeterminate thyroid nodules. Endocrine 57(2), 256-261 (2017). https://doi.org/10.1007/ s12020-016-1148-6 